\title{
The Density and Temperature of Molecular Clouds in M33
}

\author{
Christine D. Wilson ${ }^{1}$ \\ Constance E. Walker² \\ Michele D. Thornley ${ }^{3}$ \\ Submitted to the Astrophysical Journal: July 23, 1996 \\ Revised: January 21, 1996
}

Received __ ; accepted _

\footnotetext{
${ }^{1}$ Department of Physics and Astronomy, McMaster University, Hamilton, Ontario L8S 4M1 Canada

${ }^{2}$ Department of Astronomy, University of Arizona, Tucson AZ 85721 U.S.A.

${ }^{3}$ Department of Astronomy, University of Maryland, College Park, MD 20742 U.S.A.
} 


\begin{abstract}
We have observed the ${ }^{12} \mathrm{CO} \mathrm{J}=2-1,{ }^{13} \mathrm{CO} \mathrm{J}=2-1$, and ${ }^{12} \mathrm{CO} \mathrm{J}=3-2$ lines in a sample of seven giant molecular clouds in the Local Group spiral galaxy M33 using the James Clerk Maxwell Telescope. The ${ }^{12} \mathrm{CO} /{ }^{13} \mathrm{CO} \mathrm{J}=2-1$ line ratio is constant across the entire sample, while the observed ${ }^{12} \mathrm{CO} \mathrm{J}=3-2 / \mathrm{J}=2-1$ line ratio has a weak dependence on the star formation environment of the cloud, with large changes in the line ratio seen only for clouds in the immediate vicinity of an extremely luminous HII region. A large velocity gradient analysis indicates that clouds without HII regions have temperatures of $10-20 \mathrm{~K}$, clouds with HII regions have temperatures of $15-100 \mathrm{~K}$, and the cloud in the giant HII region has a temperature of at least $100 \mathrm{~K}$. Interestingly, the giant HII region appears capable of raising the kinetic temperature of the molecular gas only for clouds that are quite nearby $(<100 \mathrm{pc})$. The continuous change of physical conditions across the observed range of star formation environments suggests that the unusual physical conditions in the cloud in the giant HII region are due to post-star formation changes in the molecular gas, rather than intrinsic properties of the gas related to the formation of the giant HII region. The results from this study of M33 suggest that similar observations of ensembles of giant molecular clouds in more distant normal spiral galaxies are likely to give meaningful measurements of the average physical conditions inside the molecular clouds. These results also imply that clouds with a factor of three difference in metallicity have similar density and temperature, which in turn imply that the differences in the CO-to- $\mathrm{H}_{2}$ conversion factor seen in these clouds can be attributed entirely to metallicity effects.
\end{abstract}

Subject headings: HII regions - galaxies: individual (M33) - galaxies: ISM - 
galaxies: Local Group - ISM: individual (NGC 604) - ISM: molecules - stars: formation 


\section{Introduction}

Determining the physical conditions inside molecular clouds is important for understanding the link between the properties of the molecular gas and the types and amount of stars that are formed. Cloud properties that could affect the star formation process include the temperature and density of the molecular gas, as well as the mass fraction in high density gas. For example, higher gas temperatures might be required to form high-mass stars (Turner 1984), while a cloud with a higher mass fraction of dense gas might form stars with a higher efficiency (e.g. Lada et al. 1991). In return, star formation, particularly massive star formation, can affect conditions inside molecular clouds by compressing the gas at the boundaries of stellar wind or supernova shocks and heating the gas by increasing the ultraviolet radiation field. Giant HII regions are particularly interesting targets because they concentrate many hundreds of OB stars in a relatively small volume of space and represent the only nearby prototypes for the extreme star formation conditions in starburst and ultraluminous galaxies.

In our own Galaxy, observations of low-luminosity HII regions suggest that single high-mass stars can form from relatively low-mass molecular clouds (Hunter \& Massey 1990). Observations of several nearby giant molecular clouds show significant differences in the spatial distribution of star formation. In the Taurus-Auriga region, star formation occurs throughout the cloud as single stars or in small clusters (Kenyon et al. 1990), while Orion B has most of its star formation occurring in four massive dense cores (Lada et al. 1991), and Orion A and Ophiuchus are each forming most of their stars in a single dense cluster (McCaughrean \& Stauffer 1994, Wilking, Lada, \& Young 1989). Despite the differences in the spatial distribution of the young stars, the star formation efficiency is 1-4\% for all four regions (Evans \& Lada 1991). Interestingly, the star formation efficiency in two giant HII regions in M33 is also in the range of 2-5\% (Wilson \& Matthews 1995). 
The clumpy structure of molecular clouds (e.g. Stutzki \& Güsten 1990) implies that the volume-averaged density of a cloud is not a good measure of the density of the molecular gas producing most of the observed emission. For extragalactic molecular clouds, their low and usually unknown filling factor within the beam also implies that the kinetic temperature cannot be estimated from the peak brightness temperature of the observed line. Instead, the density and temperature must be determined using radiative transfer models and observations of many line ratios of $\mathrm{CO}$ and its isotopomers. Because this technique requires observations of some of the rarer isotopomers such as ${ }^{13} \mathrm{CO}$, it was first applied to starburst galaxies with strong CO lines (e.g. Tilanus et al. 1991, Güsten et al. 1993). However, one difficulty with even the nearest starburst galaxies is that the observations measure the emission from many molecular clouds within a single beam. For example, at a distance of $3 \mathrm{Mpc}$, a 20" beam subtends a diameter of $290 \mathrm{pc}$, which is much larger than the 10-100 pc diameter of giant molecular clouds in the Milky Way (Sanders, Scoville, \& Solomon 1985). If the physical conditions in molecular clouds vary significantly from one cloud to the next, the results of fitting the average line emission may bear little resemblance to even the average properties of the molecular cloud ensemble.

Local Group galaxies offer an advantage for such studies because they are close enough (50 kpc - $1 \mathrm{Mpc}$ ) that it is possible to isolate individual molecular clouds within a 15-20" beam. In addition, individual clouds can cover a substantial fraction of the beam and thus the $\mathrm{CO}$ lines are reasonably strong. Observations of a large sample of individual clouds can identify variations in the density and temperature from one cloud to the next, as well as correlations in cloud properties with the presence and intensity of massive star formation. The uniformity (or lack thereof) of the molecular cloud population also provides a means to test the reliability of radiative transfer techniques for determining density and temperature in more distant galaxies, where only the average emission from the molecular cloud population can be observed. In addition, density and temperature measurements 
for individual clouds can be used to assess whether changes in the density and brightness

temperature of the gas are likely to produce systematic errors in the observed correlation of the CO-to- $\mathrm{H}_{2}$ conversion factor with metallicity (Wilson 1995, Dickman, Snell, \& Schloerb 1986, Sakamoto 1996).

In this paper we present observations of seven giant molecular clouds in the spiral galaxy M33. The clouds were chosen to cover a wide variety of star formation conditions, from clouds with no optical HII regions to a cloud located in the brightest giant HII region in the galaxy. The clouds also cover a range of three in oxygen abundance (Vilchez et al. 1988). The observations and data reduction are discussed in $\S 2$. The observed line ratios are compared with previous Galactic and extragalactic observations and with the star formation environment of the clouds in $\S 3$. The density and temperature obtained from an analysis of the line ratios with a large velocity gradient code are presented in $\S 4$. The implications of the results for observations of more distant galaxies and the calibration of the CO-to- $\mathrm{H}_{2}$ conversion factor as a function of metallicity are discussed in $\S 5$. The paper is summarized in $\S 6$.

\section{Observations and Data Reduction}

Seven giant molecular clouds in M33 were observed with the James Clerk Maxwell Telescope $(\mathrm{JCMT})$ in the ${ }^{12} \mathrm{CO} \mathrm{J}=2-1,{ }^{13} \mathrm{CO} \mathrm{J}=2-1$, and ${ }^{13} \mathrm{CO} \mathrm{J}=3-2$ rotational transitions over four separate observing runs (1993 July 30 - August 1; 1993 August 25-26; 1994 July 16-18; and 1995 July 31 - August 1). The half-power beamwidth of the JCMT is $22^{\prime \prime}$ at $230 \mathrm{GHz}$ and $15^{\prime \prime}$ at $345 \mathrm{GHz}$. The clouds were selected from the interferometric samples of Wilson \& Scoville $(1990,1992)$ and have also been observed in the ${ }^{12} \mathrm{CO}$ and ${ }^{13} \mathrm{CO}$ $\mathrm{J}=1-0$ lines with 55" resolution (Wilson \& Walker 1994). One position (MC 1) includes emission from two other clouds near the half-power point of the $22^{\prime \prime}$ beam (MC 2, MC 
4, Wilson \& Scoville 1990), while only a single cloud produces the CO emission at the other positions. Each cloud was observed at a single position in the ${ }^{12} \mathrm{CO} J=2-1$ and ${ }^{13} \mathrm{CO}$ $\mathrm{J}=2-1$ lines and in a five-point cross spaced by 8 " in the ${ }^{12} \mathrm{CO} \mathrm{J}=3-2$ line. All observations were obtained by position-switching to a location in the inner galaxy $(\alpha(1950)=01: 31: 16.9$, $\delta(1950)=30: 24: 54)$ that had been determined previously to be free of ${ }^{12} \mathrm{CO} \mathrm{J}=1-0$ and $\mathrm{J}=2-1$ emission. Observations of the ${ }^{13} \mathrm{CO} \mathrm{J}=2-1$ line were obtained at two or more different velocities per source to avoid the problem of spikes in the spectrometer masquerading as a weak line. Except for the observations in 1993 July, which were obtained with the Canadian Acousto-Optical Spectrometer, all observations were obtained using the Dutch Autocorrelation Spectrometer. A log of the observations is given in Table 1 .

The calibration was monitored by frequently observing both planets and spectral line calibrators. The main beam efficiencies determined by observing Saturn, Mars, and Jupiter during the runs in 1993 and 1995 agreed well with the published main beam efficiencies, and thus for these runs we adopt the main beam efficiencies from the JCMT User's Guide of 0.69 at 220 and $230 \mathrm{GHz}$ and 0.58 at $345 \mathrm{GHz}$. The spectral line calibrators were the evolved stars CRL 2688, CRL 618, and the compact HII region NGC 7538 IRS1. The calibrator observations agree very well with reference spectra available at the JCMT, with an average ratio of the observed to reference peak temperature of 1.00 with an rms scatter of $13 \%$. The rms dispersion of the individual measurements is $10 \%$ for the ${ }^{12} \mathrm{CO}$ observations and $20 \%$ for the ${ }^{13} \mathrm{CO}$ observations. We attribute the larger scatter in the ${ }^{13} \mathrm{CO}$ observations to the weakness of the lines and adopt $10 \%$ as our absolute calibration uncertainty in any single line on the basis of the ${ }^{12} \mathrm{CO}$ observations.

Throughout the 1994 run, the peak temperatures observed for CRL 618 and CRL 2688 were consistently $70 \%$ of the values measured in the other runs. The low efficiency of the telescope was confirmed by remeasuring the ${ }^{12} \mathrm{CO} \mathrm{J}=2-1$ spectrum of $\mathrm{MC} 20$, which 
was also $70 \%$ of the previously measured value. The main beam efficiency determined from observations of Mars and Uranus was also low by the same amount. Thus for the data obtained during this run, we adopt a main beam efficiency at 220 and $230 \mathrm{GHz}$ of $0.70 \times 0.69=0.48$.

As we are comparing line strengths measured with the same beam diameter (either the original beam or convolved to a larger beam), the best temperature scale to use would be the $T_{R}^{*}$ scale. Although the clouds in M33 are much smaller than the full diffraction pattern of the telescope $\left(5-16^{\prime \prime}\right)$, the $T_{R}^{*}$ scale corrects for all telescope efficiencies except the source-beam coupling efficiency, which should be the same at all frequencies since we have observed the emission with the same beam at each frequency. However, to convert the observed temperatures $T_{A}^{*}$ to $T_{R}^{*}$ requires knowing $\eta_{f s s}$, the forward scattering and spillover efficiency. This efficiency is difficult to measure, and so we did not attempt to measure $\eta_{f s s}$ during our observing runs. As a result, we do not know how to correct the published values of $\eta_{f s s}$ for the calibration problems encountered in the 1994 run. However, we do have good measurements of the main beam efficiency for each run and under normal calibration conditions, adopting the main beam temperature scale would only change the ${ }^{12} \mathrm{CO} \mathrm{J}=3-2 / \mathrm{J}=2-1$ line ratios by $5 \%$ compared to the $T_{R}^{*}$ scale. Thus we use the main beam temperature scale throughout this paper, and in particular in calculating the line ratios for comparison with radiative transfer models.

Given the angular size of the M33 molecular clouds (5-16"), the main beam temperature, $T_{M B}$, is a better approximation to the brightness or radiation temperature, $T_{R}$, than is the antenna temperature, $T_{A}^{*}$. The three temperature scales are related by

$$
T_{R}=\frac{T_{A}^{*}}{\eta_{c} \eta_{f s s}}=\frac{T_{M B} \eta_{M B}}{\eta_{c} \eta_{f s s}}
$$

where $\eta_{c}$ is the source-beam coupling factor, $\eta_{f s s}$ is the forward scattering and spillover efficiency, and $\eta_{M B}$ is the main beam efficiency. We can estimate $\eta_{c}$ for the M33 clouds since 
they have been observed at high resolution (Wilson \& Scoville 1990, 1992). For the $230 \mathrm{GHz}$ observations, $\eta_{c}>0.9$, while $\eta_{f s s}=0.80$ and $\eta_{M B}=0.69$, so that $T_{R} \sim 0.9 T_{M B}-1.0 T_{M B}$. At $345 \mathrm{GHz}, \eta_{c}>0.8$, while $\eta_{f s s}=0.70$ and $\eta_{M B}=0.58$, so that $T_{R} \sim 0.8 T_{M B}-1.0 T_{M B}$. We use the main beam temperature scale throughout this paper, and in particular in calculating the line ratios for comparison with radiative transfer models.

The data were reduced using the Bell Labs data reduction package COMB. The data were binned to a resolution of $1 \mathrm{~km} \mathrm{~s}^{-1}$ and first to third order baselines removed. The lines are quite narrow $\left(\sim 20 \mathrm{~km} \mathrm{~s}^{-1}\right)$ and the spectrometer bandwidth was always at least $200 \mathrm{~km} \mathrm{~s}^{-1}$, so the higher order baselines should not introduce significant errors into the final line intensities. Each set of five ${ }^{12} \mathrm{CO} \mathrm{J}=3-2$ spectra were then convolved to simulate a 22" beam. The spectra for each cloud are shown in Figure 11. Channel-by-channel ratios of the two spectra were used to measure the average line ratio for each cloud. Only channels with a signal-to-noise ratio of at least 2 in the line ratio were used. This restriction resulted in the ${ }^{12} \mathrm{CO} /{ }^{13} \mathrm{CO} \mathrm{J}=2-1$ line ratio being obtained from the central $5-14 \mathrm{~km} \mathrm{~s}^{-1}$ $\left(0.6-1.3 \times \Delta V_{F W H M}\right)$, while the ${ }^{12} \mathrm{CO} \mathrm{J}=3-2 / \mathrm{J}=2-1$ line ratio was obtained from the central 9-19 $\mathrm{km} \mathrm{s}^{-1}\left(1.1-1.7 \times \Delta V_{F W H M}\right)$. The measured line ratios and ${ }^{12} \mathrm{CO} \mathrm{J}=2-1$ integrated intensity for each cloud are given in Table 2 .

\section{CO Line Ratios and the Star Formation Environment}

The ${ }^{12} \mathrm{CO} /{ }^{13} \mathrm{CO} \mathrm{J}=2-1$ line ratios given in Table 2 have an average value of 7.3 and an rms dispersion of $1.3(18 \%)$. Thus we conclude that there is no intrinsic variation in the ${ }^{12} \mathrm{CO} /{ }^{13} \mathrm{CO} \mathrm{J}=2-1$ line ratio from one cloud to another in M33. This average line ratio compares quite well with values measured in other galaxies such as M51 (6-10 in

center and spiral arms, Garcia-Burillo, Guelin, \& Cernicharo 1993) and NGC 4414 (9 \pm 1 , Braine, Combes, \& van Driel 1993). The value in M33 also agrees quite well with the 
value measured in a pencil-beam survey of the Milky Way (5.5 \pm 1 ,Sanders et al. 1993) and in large-area maps of the Orion A and B molecular clouds (4-6 along the main ridge, Sakamoto et al. 1994). The M33 value is slightly smaller than the value measured in the starburst galaxy M82 (12 \pm 3, Tilanus et al. 1991) and in a large sample of starburst and interacting galaxies (13 \pm 5 , Aalto et al. 1995). Previous measurements have indicated that the ${ }^{12} \mathrm{CO} /{ }^{13} \mathrm{CO} \mathrm{J}=1-0$ line ratio is also somewhat larger in starburst and merging galaxies compared with other galaxies (Becker \& Freudling 1991; Aalto et al. 1991; Combes et al. 1991; Casoli et al. 1991).

The ${ }^{12} \mathrm{CO} \mathrm{J}=3-2 / \mathrm{J}=2-1$ line ratios given in Table 2 show more scatter. In particular, the line ratio obtained for NGC 604-2 $(1.07 \pm 0.03$ is significantly higher than the value for any other cloud. Excluding NGC 604-2, the average ${ }^{12} \mathrm{CO} \mathrm{J}=3-2 / \mathrm{J}=2-1$ line ratio is 0.69 with an rms dispersion of $0.15(21 \%)$. This average line ratio for the six clouds in M33 is in good agreement with values measured in other galaxies such as the starburst galaxies M82 (0.8 \pm 0.2, Güsten et al. 1993), NGC 253 (0.5 \pm 0.1 in the disk, Wall et al. 1991), and IC $342(0.47 \pm 0.12$, Irwin \& Avery 1993). The average value for the six clouds in M33 also agrees well with values measured in M51 (0.7 in the spiral arms, Garcia-Burillo et al. 1993) and in a pencil-beam survey of the Milky Way (0.55 \pm 0.08 , Sanders et al. 1993). It also agrees with a previous measurement for M33 in a slightly larger beam $(0.64 \pm 0.28$, Thornley \& Wilson 1994). Higher line ratios similar to the value for the cloud NGC 604-2 are seen in the nuclei of both NGC 253 ( 1, Wall et al. 1991) and M51 (1.1, Garcia-Burillo et al. 1993).

The clouds observed in M33 have oxygen abundances that range over a factor of three (Vilchez et al. 1988). If we momentarily ignore the unusually high ${ }^{12} \mathrm{CO} \mathrm{J}=3-2 / 2-1$ line ratio in NGC 604-2, there is no evidence that the observed line ratios depend on the metallicity of the cloud. In particular, the two line ratios for MC 19 and NGC 604-4 are 
very similar despite a factor of three difference in the oxygen abundance (Table 2). NGC 604-2 and NGC 604-4 are located within 120 pc of each other and presumably share the same oxygen abundance, so we attribute the difference in the ${ }^{12} \mathrm{CO} \mathrm{J}=3-2 / 2-1$ line ratios for these two clouds to some factor other than metallicity, most likely the star formation environment.

If we examine the star formation environments of our cloud sample, two of the clouds (MC 19 and MC 32) lie between 100 and 150 pc away from the nearest optical HII region (Wilson \& Scoville 1991), while three clouds (MC 1, MC 13, and MC 20) contain optical HII regions. The cloud NGC 604-2 is located near the center of the giant HII region NGC 604 , on the southern edge of the OB association which presumably powers the HII region (Wilson \& Matthews 1995), while the cloud NGC 604-4 is located at the very edge of the southern extent of the $\mathrm{H} \alpha$ emission from the HII region. If we compare the $\mathrm{CO}$ line ratios for the three clouds without HII regions (MC 19, MC 32, and NGC 604-4) with those for the clouds with normal HII regions ( $\mathrm{MC} 1, \mathrm{MC} 13$, and $\mathrm{MC} 20$ ), the average ${ }^{12} \mathrm{CO} /{ }^{13} \mathrm{CO}$ $\mathrm{J}=2-1$ line ratios for the two sets of clouds agree very well. The ${ }^{12} \mathrm{CO} \mathrm{J}=3-2 / \mathrm{J}=2-1$ line ratios for the clouds with optical HII regions $(0.79 \pm 0.05)$ are somewhat higher than those for the clouds without optical HII regions $(0.58 \pm 0.06)$, while the line ratio for NGC 604-2 in the giant HII region is even higher $(1.07 \pm 0.03)$. The difference between the two clouds (NGC 604-2 and NGC 604-4) in and around the giant HII region is striking. The projected separation of the two clouds is only $120 \mathrm{pc}\left(30^{\prime \prime}\right)$, yet only the cloud in the immediate vicinity of the giant HII region and its powering OB association has an unusual line ratio.

The high ${ }^{12} \mathrm{CO} \mathrm{J}=3-2 / \mathrm{J}=2-1$ line ratio in NGC $604-2$ may be a clue to unusual physical conditions in the molecular clouds from which the HII region NGC 604 formed, i.e. a pre-star formation difference in the physical conditions that may have caused the formation of the giant HII region. Alternatively, the high line ratio may be due to heating of the 
gas by the massive stars, i.e. a post-star formation change in the physical conditions in the molecular cloud. This interpretation implies that the giant HII region has a relatively small sphere of influence over which its intense radiation field can change the properties of the dense molecular gas. It appears that both an unusually intense radiation field and a cloud in close proximity to the source of the ionizing radiation are required to produce a large change in the $\mathrm{CO}$ line ratios. However, the ${ }^{12} \mathrm{CO} \mathrm{J}=3-2 / \mathrm{J}=2-1$ line ratios observed for the clouds with optical HII regions are higher than those for clouds without HII regions at the $2 \sigma$ level. This difference suggests that HII regions that are more than an order of magnitude less luminous than NGC 604 can produce smaller changes in the CO line ratios. Thus the data for M33 provide some evidence for a continuous change of line ratio with increasing intensity in the star formation environment.

One complication to this interpretation is that the optical HII regions could in principle be separated from the molecular cloud by up to the scale height of the disk $(\sim 200 \mathrm{pc})$ and simply appear projected on the cloud. However, the fact that most HII regions appear close to molecular clouds (Wilson \& Scoville 1991) suggests that there is a true physical association between the clouds and the HII regions. Also, the three clouds without optical HII regions could actually be forming stars, since the clouds have sufficient optical depths to hide an HII region if it were located on the far side of the cloud. However, since the ${ }^{12} \mathrm{CO}$ lines are optically thick for these clouds, we can only see emission from the near and presumably cool side of the cloud and so whether or not the clouds have an HII region on their far side is irrelevant to this analysis. The increasing line ratio with increasing HII region luminosity is consistent with a picture in which the ultraviolet radiation field of the optically visible HII region heats the molecular gas. We conclude that the ${ }^{12} \mathrm{CO}$ $\mathrm{J}=3-2 / \mathrm{J}=2-1$ line ratio of a cloud has a weak dependence on the star formation environment of the cloud, with large changes in the line ratio seen only for clouds in the immediate vicinity of an extremely luminous HII region. 


\section{Density and Temperature Determined from Large Velocity Gradient Models}

\subsection{The Large Velocity Gradient Model}

There are several different assumptions that can be made in calculating source parameters from molecular line data. The most common assumption is that the gas is in local thermodynamic equilibrium (LTE), where the number density of molecules in any particular energy level is only a function of temperature. Probably the next most common assumption is that there is a large velocity gradient (LVG) in the gas cloud (e.g. Scoville \& Solomon 1974). The presence of such a gradient has the effect of Doppler-shifting the emission of a molecule relative to that of molecules elsewhere in the cloud. When the mean free path of a line photon exceeds the local velocity dispersion divided by the velocity gradient, it can travel through the cloud without being absorbed elsewhere. A third approach is to assume that the velocity field is microturbulent. In microturbulent radiative transfer models, the velocity structure is due to thermal motions and small-scale random motions (White 1977). Unlike the LVG case, neighboring cloud components are radiatively coupled, with the degree to which they are coupled depending on the magnitude of the random motions and the amount of resonance scattering. A fourth possibility is to use a photon mean escape probability formalism to simplify radiative transfer calculations. In this approach, level populations and the internal intensity are calculated for an "average"

location in a uniform, spherical cloud, with the photon escape probability from this position being determined by the optical depth (Black \& Aalto 1991, private communication). These latter three non-LTE techniques often yield similar results when applied to galactic molecular clouds (White 1977) and thus we have chosen to use the LVG formalism in this analysis.

To estimate the physical conditions in the molecular clouds in M33, we used the 
large velocity gradient code RAD written by Lee Mundy and implemented as part of the MIRIAD data reduction package. Models were run for kinetic temperatures $T_{K}=10,15,20,30,50,100,200,300 \mathrm{~K}$ and for three different values of the $\left[{ }^{12} \mathrm{CO}\right] /\left[{ }^{13} \mathrm{CO}\right]$ abundance ratio $(30,50,70)$ to span the range of abundances seen in the Milky Way (Langer \& Penzias 1990). For each kinetic temperature and abundance combination, the $\mathrm{H}_{2}$ density range was $n_{H_{2}}=10-10^{6} \mathrm{~cm}^{-3}$ and the ${ }^{12} \mathrm{CO}$ column density range per unit velocity was $N\left({ }^{12} \mathrm{CO}\right) / \Delta \mathrm{V}=10^{15}-10^{20} \mathrm{~cm}^{-2}\left(\mathrm{~km} \mathrm{~s}^{-1}\right)^{-1}$. We use the full-width half maximum velocity to calculate the ${ }^{12} \mathrm{CO}$ column density from $N / \Delta V$, where the average velocity width for the six clouds excluding NGC $604-2$ is $9.3 \pm 0.6 \mathrm{~km} \mathrm{~s}^{-1}$.

One drawback of both the LTE and non-LTE techniques described here is that they do not take into account the effects of cloud-cloud shielding. Cloud-cloud shielding occurs when two or more molecular clouds are aligned along our line of sight in both space and velocity. In this case the radiation from the background cloud(s) may be fully or partially absorbed by the foreground cloud. When a pair of isotopes, such as ${ }^{12} \mathrm{CO}$ and ${ }^{13} \mathrm{CO}$, are being used to probe the optical depth along a line of sight through a galaxy, cloud-cloud shielding will tend to produce overestimates of the optical depth in individual clouds. Optical depth estimates through the ensemble of clouds are only affected by cloud-cloud shielding when the rarer isotope (e.g. $\left.{ }^{13} \mathrm{CO}\right)$ becomes optically thick and so does not sample all the clouds along the line of sight. Cloud-cloud shielding is unlikely to be a significant problem in M33, which has a relatively low molecular gas surface density and is not highly inclined.

\subsection{Model Results for M33 Molecular Clouds}

To provide better constraints for the models, we include the ${ }^{12} \mathrm{CO} /{ }^{13} \mathrm{CO} \mathrm{J}=1-0$ line ratio observed for these clouds in a 55" beam (Wilson \& Walker 1994). (The possibility that the larger beam size of the $J=1-0$ data can produce systematic errors in our results is 
discussed later in this section.) Although both the NGC 604 clouds were observed within a single beam, we can use their significantly different central velocities to isolate the line ratio for each cloud. The average ${ }^{12} \mathrm{CO} /{ }^{13} \mathrm{CO} \mathrm{J}=1-0$ line ratio for the seven clouds is $9.7 \pm 0.6$ with an rms dispersion of 1.5 or $15 \%$. We combine this average $\mathrm{J}=1-0$ line ratio with the average $\mathrm{J}=2-1$ line ratio in our modeling. Since there appears to be a trend in the ${ }^{12} \mathrm{CO}$ $\mathrm{J}=3-2 / \mathrm{J}=2-1$ line ratio with star formation environment, we used four values of this line ratio in our analysis: the average value for all six clouds $(0.69 \pm 0.06)$; the average value for clouds without HII regions $(0.58 \pm 0.06)$; the average value for clouds with HII regions $(0.79 \pm 0.05)$; and the value for NGC 604-2 (1.07 \pm 0.16$)$, where the total uncertainty in the line ratio for NGC 604-2 has been estimated at $15 \%$ to match the expected calibration uncertainty in the line ratio.

Acceptable solutions for each set of three line ratios $\left({ }^{12} \mathrm{CO} /{ }^{13} \mathrm{CO} \mathrm{J}=1-0,{ }^{12} \mathrm{CO} /{ }^{13} \mathrm{CO}\right.$ $\mathrm{J}=2-1$, and ${ }^{12} \mathrm{CO} \mathrm{J}=3-2 / \mathrm{J}=2-1$ ) were found by visual inspection of plots of the line ratios as a function of density and column density at each kinetic temperature. Acceptable solutions were defined to be ones for which all three line ratios agreed within their $1 \sigma$ uncertainties. Examples of representative solutions are given in Figure 2 and the full range of solutions is given in Table 3. The ranges of values given for temperature, density, and column density for each $\left[{ }^{12} \mathrm{CO}\right] /\left[{ }^{13} \mathrm{CO}\right]$ abundance ratio are correlated in the sense that solutions with higher temperatures have higher column densities and lower densities.

The results from the LVG analysis show that the kinetic temperature increases from clouds without HII regions $\left(T_{K}=10-20 \mathrm{~K}\right)$ to clouds with HII regions $\left(T_{K}=15-100\right.$ K) to NGC $604-2\left(T_{K} \geq 100 \mathrm{~K}\right)$. The column density also increases by at least an order of magnitude from clouds without HII regions to NGC 604-2. The molecular gas in all seven clouds is quite dense $\left(10^{3}-3 \times 10^{4} \mathrm{~cm}^{-3}\right)$, although the density is lower in NGC 604-2 than in the clouds without HII regions. Thus an increase in the ${ }^{12} \mathrm{CO} \mathrm{J}=3-2 / \mathrm{J}=2-1$ line ratio 
produces LVG solutions with higher kinetic temperatures, higher column densities, and lower densities. The volume-averaged densities (mass divided by volume) are $40-210 \mathrm{~cm}^{-3}$ for the three clouds without HII regions and $\sim 200-400 \mathrm{~cm}^{-3}$ for the three clouds with HII regions (calculated from Wilson \& Scoville 1990, 1992), significantly lower than the densities derived from the LVG analysis $\left(5 \times 10^{3}-3 \times 10^{4} \mathrm{~cm}^{-3}\right.$ and $2 \times 10^{3}-10^{4} \mathrm{~cm}^{-3}$, respectively). The volume filling factor of the dense gas is $0.1-4 \%$ for clouds without HII regions and $\sim 2-20 \%$ for clouds with HII regions. For NGC 604-2 the volume-averaged density is somewhat higher, $\sim 500 \mathrm{~cm}^{-3}$, which combined with the somewhat lower density from the LVG model of $10^{3}-3 \times 10^{3} \mathrm{~cm}^{-3}$ gives a filling factor for the dense gas of $17-50 \%$. These results compare well with the volume filling factor of $10 \%$ for clumps in Orion (Sakamoto et al. 1994).

Are the unusual physical conditions in NGC 604-2 due to the presence of the giant HII region, or do they represent pristine conditions in the molecular gas that could have played a role in initiating the formation of the giant HII regions? An increase in the kinetic temperature of the gas could be easily accomplished by the intense ultraviolet radiation field produced by the dense cluster of OB stars powering the giant HII region. The gas column density could be increased through shock-initiated merging of two or more molecular clouds or through a partial collapse of the molecular cloud due to an increase in the external gas pressure. However, the molecular column density could also be decreased through photo-dissociation of the molecular gas and thus it is difficult to predict the net effect on the column density from the formation of the giant HII region. It is difficult to understand how the formation of the giant HII region could have produced the higher filling factor and lower density of the dense clumps in the molecular cloud. One possible scenario would be that the formation of the dense star cluster depleted the gas in the densest clumps, leaving behind the lower density inter-clump gas. This gas could then be compressed to somewhat higher density as the cloud shrank due to the increased external gas pressure. In any 
case, the continuous range of all physical conditions (kinetic temperature, column density, density, and filling factor) observed from clouds without HII regions to clouds with normal HII regions to this cloud in a giant HII region suggests that the different physical conditions in NGC 604-2 are due to post-star formation changes in the molecular gas, rather than intrinsic conditions of the pre-star formation molecular cloud.

The similarity of the $\mathrm{CO}$ line ratios for the six clouds in the normal disk despite a range of a factor of three in oxygen abundance might naively suggest that the $\left[{ }^{12} \mathrm{CO}\right] /\left[{ }^{13} \mathrm{CO}\right]$ abundance ratio does not change significantly when the oxygen abundance is varied by a factor of three. However, this conclusion is in contrast to the Milky Way, where Langer \& Penzias (1990) have measured a significant radial gradient in the $\left[{ }^{12} \mathrm{CO}\right] /\left[{ }^{13} \mathrm{CO}\right]$ abundance ratio. In fact, for kinetic temperatures of 10-20 K there do exist possible LVG solutions with different values for the $\left[{ }^{12} \mathrm{CO}\right] /\left[{ }^{13} \mathrm{CO}\right]$ abundance ratio but with the same density, kinetic temperature, and ${ }^{13} \mathrm{CO}$ column density which produce identical CO line ratios (Figure 31). This result can be easily understood as being due to the low optical depth of the ${ }^{13} \mathrm{CO}$ emission and the high optical depth of the ${ }^{12} \mathrm{CO}$ emission. As long as the ${ }^{13} \mathrm{CO}$ column density is fixed, the observed line ratios will be the same for a large range in the $\left[{ }^{12} \mathrm{CO}\right] /\left[{ }^{13} \mathrm{CO}\right]$ abundance ratio. Thus our data are consistent with M33 containing a similar radial gradient in the $\left[{ }^{12} \mathrm{CO}\right] /\left[{ }^{13} \mathrm{CO}\right]$ abundance ratio as found in the Milky Way, without requiring any conspiracy between several physical parameters to produce the observed constant CO line ratios.

Using the average ${ }^{12} \mathrm{CO} \mathrm{J}=3-2 / \mathrm{J}=2-1$ line ratio for the six clouds (excluding NGC 604-2) in the LVG analysis produces solutions for temperature, density, and column density that are intermediate between those obtained for the clouds with and without HII regions separately (Table 3). The mean value for each parameter derived using the average line ratio is within a factor of two of the average of the mean parameters derived for clouds 
with and without HII regions separately. Thus physical conditions derived by averaging together emission from clouds both with and without HII regions provide a reasonable approximation to the average physical conditions in this group of six molecular clouds.

\subsection{Possible Sources of Systematic Errors}

There are several possible complications that could introduce systematic errors into the LVG results. For example, the difference in the beam area used in the ${ }^{12} \mathrm{CO} /{ }^{13} \mathrm{CO}$ $\mathrm{J}=1-0$ ratio compared with the other ratios could introduce systematic errors if the $\mathrm{J}=1-0$ line ratio varies as a function of the spatial resolution used (Wilson \& Walker 1994). We can assess what effect this would have by looking at the LVG results using a lower value of $5 \pm 1$, which is similar to the values of 3 to 6 seen in Galactic molecular clouds (Gordon \& Burton 1976, Solomon, Scoville, \& Sanders 1979). For simplicity we will use the results for the average of all six clouds in this analysis. For a $\left[{ }^{12} \mathrm{CO}\right] /\left[{ }^{13} \mathrm{CO}\right]$ abundance ratio of 50 and this lower $\mathrm{J}=1-0$ line ratio, the solutions permitted for the six clouds are $T_{K}=20-300 \mathrm{~K}$, $n=60-2000 \mathrm{~cm}^{-3}$, and $N\left({ }^{12} \mathrm{CO}\right)=9 \times 10^{17}-3 \times 10^{19} \mathrm{~cm}^{-2}$. The solutions permitted for NGC 604-2 are $T_{K}=50-300 \mathrm{~K}, n=30-70 \mathrm{~cm}^{-3}$, and $N\left({ }^{12} \mathrm{CO}\right)=1-2 \times 10^{19} \mathrm{~cm}^{-2}$, with an additional possible solution with $T_{K}=300 \mathrm{~K}, n=600 \mathrm{~cm}^{-3}$, and $N\left({ }^{12} \mathrm{CO}\right)=2 \times 10^{19}$ $\mathrm{cm}^{-2}$. Much of the increased range of permitted solutions can be attributed to the larger uncertainty in the lower adopted line ratio (20\%) compared with the observed line ratio $(6 \%)$. On average, the effect of adopting this lower $\mathrm{J}=1-0$ line ratio is to increase the kinetic temperature range for which solutions are available, to decrease the density by a factor varying from a few to more than an order of magnitude, and to increase the column density and its range.

Yet another possible difficulty is that the sizes of the clouds may be different in the different transitions. In applying the LVG analysis we have implicitly assumed that the 
beam filling factor is the same in each line. Recent observations of the Orion molecular cloud show that the cloud appears smaller in the ${ }^{12} \mathrm{CO} J=2-1$ line than in the $\mathrm{J}=1-0$ line, while the ${ }^{13} \mathrm{CO} J=2-1$ line may have an even narrower extent (Sakamoto et al. 1994). If the $\mathrm{J}=2-1$ and $\mathrm{J}=3-2$ emission regions have different angular extents, we would expect the $\mathrm{J}=3-2$ emission to originate in the smaller region. In this case, the observed ${ }^{12} \mathrm{CO}$ $\mathrm{J}=3-2 / \mathrm{J}=2-1$ line ratios would set only lower limits on the true line ratios, and so the true kinetic temperatures would be larger than those derived here. In this scenario, the large ${ }^{12} \mathrm{CO} \mathrm{J}=3-2 / \mathrm{J}=2-1$ line ratio in NGC 604-2 could be due to a larger ratio of the $\mathrm{J}=3-2$ to $\mathrm{J}=2-1$ emitting areas compared with the other clouds. These hypotheses could be tested with an imaging submillimeter interferometer such as the Smithsonian Astrophysical Observatory's Submillimeter Array, which is presently under construction on Mauna Kea.

Another possible problem is that more than one temperature component or a smooth range of temperatures may be present in some or all of the clouds in our sample (for example, see models by Gierens, Stutzki, \& Winnewisser 1992). This is particularly true for NGC 604-2, which, depending on its three-dimensional orientation relative to the giant HII region, could be subject to intense heating on only one side. Some support for a two-component model is given by the brightness temperatures predicted by models with $T_{K} \geq 100 \mathrm{~K}$. The brightness temperature predicted for the ${ }^{12} \mathrm{CO} \mathrm{J}=2-1$ line is $\geq 60$ $\mathrm{K}$, which when combined with the observed peak temperature of $1.19 \mathrm{~K}$ gives a filling factor of only $2 \%$. This filling factor corresponds to a source diameter of only $3^{\prime \prime}$ in a $22^{\prime \prime}$ beam, significantly smaller than the $9^{\prime \prime}$ diameter measured in the ${ }^{12} \mathrm{CO} \mathrm{J}=1-0$ line (Wilson \& Scoville 1992). A crude two-component model for NGC 604-2 could consist of one component with line ratios like the six other clouds and a hot component with ${ }^{12} \mathrm{CO}$ $\mathrm{J}=3-2 / 2-1 \sim 2$. This model predicts that the two components contribute about equally to the ${ }^{12} \mathrm{CO} \mathrm{J}=3-2$ emission, while roughly three-quarters of the ${ }^{12} \mathrm{CO} \mathrm{J}=2-1$ emission arises in the cooler component. This cool component gives a brightness temperature of $\sim 5 \mathrm{~K}$ 
for $T_{K}=10 \mathrm{~K}$, which corresponds to a source diameter of $\sim 9^{\prime \prime}$. Additional circumstantial

evidence in support of more than one temperature component comes from the measurement of the ${ }^{12} \mathrm{CO} \mathrm{J}=2-1 / 1-0$ line ratio in MC 32 and MC 19 (Thornley \& Wilson 1994). Despite the relatively large measurement uncertainty $\left(0.67 \pm 0.19\right.$ in a $54^{\prime \prime}$ beam), this line ratio only overlaps the solutions for the three line ratios discussed here for $T_{K}=15 \mathrm{~K}$ and $\left[{ }^{12} \mathrm{CO}\right] /\left[{ }^{13} \mathrm{CO}\right]$ abundance ratios of $50-70$. A more precise measurement of this line ratio might easily remove this area of overlap, which would again suggest that the lowest-J rotational transitions originate in a cooler component than the $\mathrm{J}=3-2$ transition.

\section{Implications for Distant Galaxies and the CO-to- $\mathrm{H}_{2}$ Conversion Factor}

The relative uniformity of the line ratios of the six molecular clouds observed in the normal disk of M33 suggests that similar observations of ensembles of molecular clouds in more distant galaxies are likely to produce meaningful measurements of the average physical conditions of the molecular gas. This conclusion is also supported by the reasonable agreement between physical conditions derived using the average line ratios for the six clouds and the average of the physical conditions derived separately for clouds with and without HII regions $(\S 3)$. The relatively small sphere of influence $(\sim 100 \mathrm{pc})$ of the giant HII region NGC 604 suggests that in normal galaxies only the most intense star forming regions may produce significant changes in the molecular gas. The change in the line ratios is likely to be measurable only in relatively nearby galaxies $(<10 \mathrm{Mpc})$, where the warm molecular gas in the HII region is not diluted by emission from cooler gas included in the beam. It would be interesting to test whether similarly uniform line ratios are observed in individual clouds in the intense ultraviolet field of a starburst galaxy, but such observations must await the construction of an imaging submillimeter interferometer.

The CO-to- $\mathrm{H}_{2}$ conversion factor, $\alpha$, is predicted to depend on the density and 
brightness temperature of the molecular clouds as $\sqrt{n} / T_{B}$, as well as on the metallicity (Dickman et al. 1986, Sakamoto 1996). Observations of Local Group galaxies, including several of these clouds in M33, have been used to calibrate the dependence of the conversion factor on metallicity (Wilson 1995). However, given the generally lower shielding by dust and higher ultraviolet radiation field in low-metallicity irregular galaxies, it is possible that molecular clouds in lower metallicity environments have systematically different brightness temperatures and densities. We can use our solutions for the physical conditions in the molecular clouds in M33 to determine the possible range in $\sqrt{n} / T_{B}$ and to see whether systematic errors in the metallicity calibration are likely to exist.

Ignoring the possible effects of density and brightness temperature differences, Wilson (1995) obtained $\alpha \propto(12+\log (O / H))^{-0.67}$. For the six molecular clouds (excluding NGC 604-2), $\sqrt{n} / T_{B}$ can vary by a factor of six for kinetic temperature solutions from 10 to 30 $\mathrm{K}$. This large range in $\sqrt{n} / T_{B}$ is produced by the tendency of the solutions to have lower densities and higher brightness temperatures for higher kinetic temperatures. As a result, it is theoretically possible for NGC 604-4, which has a metallicity three times smaller than the other five clouds, to have $\sqrt{n} / T_{B}$ a factor of six smaller, which would produce a significantly steeper slope $(\sim-2)$ in the derived metallicity calibration. However, to produce this factor of six difference in $\sqrt{n} / T_{B}$ requires NGC $604-4$ to have a $\left[{ }^{12} \mathrm{CO}\right] /\left[{ }^{13} \mathrm{CO}\right]$ abundance ratio of 30 , while the clouds in the inner disk would need an abundance ratio of 50 . Thus to produce significant systematic errors in the metallicity calibration of the CO-to- $\mathrm{H}_{2}$ conversion factor would require a radial $\left[{ }^{12} \mathrm{CO}\right] /\left[{ }^{13} \mathrm{CO}\right]$ gradient in the opposite sense from that observed in the Milky Way (Langer \& Penzias 1990). In addition, the different densities $\left(4 \times 10^{3}\right.$ $\mathrm{cm}^{-3}$ vs. $2 \times 10^{4} \mathrm{~cm}^{-3}$ ) and excitation temperatures ( $30 \mathrm{~K}$ vs. $10 \mathrm{~K}$ ) for NGC 604-4 and the inner disk clouds would have to "conspire" to produce the same observed CO line ratios despite large physical differences. We conclude that the molecular clouds in M33 provide no evidence for a systematic error in the metallicity calibration of the CO-to- $\mathrm{H}_{2}$ 
conversion factor due to differences in the density or brightness temperature from low to high metallicity clouds.

\section{Conclusions}

We have observed the ${ }^{12} \mathrm{CO} \mathrm{J}=2-1,{ }^{13} \mathrm{CO} \mathrm{J}=2-1$, and ${ }^{12} \mathrm{CO} \mathrm{J}=3-2$ lines in a sample of seven giant molecular clouds in the Local Group spiral galaxy M33. The clouds were chosen to cover a range of star formation conditions, from clouds without optical HII regions to a cloud in a giant $\mathrm{HII}$ region. We find that the ${ }^{12} \mathrm{CO} /{ }^{13} \mathrm{CO} \mathrm{J}=2-1$ line ratio is constant across the entire sample, while the ${ }^{12} \mathrm{CO} \mathrm{J}=3-2 / \mathrm{J}=2-1$ line ratio is somewhat smaller in three clouds without optical HII regions than in three clouds with HII regions. The seventh cloud, located in the brightest giant HII region in the galaxy, has an even higher ${ }^{12} \mathrm{CO}$ $\mathrm{J}=3-2 / \mathrm{J}=2-1$ line ratio. We conclude that the ${ }^{12} \mathrm{CO} \mathrm{J}=3-2 / \mathrm{J}=2-1$ line ratio of a cloud has a weak dependence on the star formation environment of the cloud, with large changes in the line ratio seen only for clouds in the immediate vicinity of an extremely luminous HII region.

We used a large velocity gradient code to determine the density, temperature, and column density for the clouds. The analysis indicates that clouds without HII regions have temperatures in the range of 10 to $20 \mathrm{~K}$ and clouds with HII regions have temperatures in the range of 15 to $100 \mathrm{~K}$, while the cloud in the giant HII region has a kinetic temperature of at least $100 \mathrm{~K}$. We note that the giant HII region seems to have a relatively limited sphere of influence within which it can heat the molecular gas, since a molecular cloud located only 120 pc from the giant HII region shows a normal cooler temperature. The column density increases by about an order of magnitude from the cool clouds to the hot cloud, as does the volume filling factor of the dense gas, while the density decreases by less than an order of magnitude. The continuity of physical properties (kinetic temperature, 
density, column density, and filling factor) across the range of star formation environments in our sample suggests that the unusual physical conditions seen in the cloud in the giant HII region are due to post-star formation changes in the molecular gas, rather than intrinsic properties of the gas related to the formation of the giant HII region.

Excluding the giant HII region, the relatively uniform line ratios observed in M33 suggest that average physical conditions determined from similar measurements of ensembles of giant molecular clouds in more distant normal spiral galaxies are likely to give physically meaningful results. These uniform line ratios also imply that the average brightness temperature and density are similar in clouds ranging over a factor of three in metallicity, which in turn suggests that differences in the CO-to- $\mathrm{H}_{2}$ conversion factor seen in these clouds can be attributed entirely to metallicity effects.

The research of CDW is supported through a grant from the Natural Sciences and Engineering Research Council of Canada. MDT thanks NRC Canada for travel support. The JCMT is operated by the Royal Observatories on behalf of the Particle Physics and Astronomy Research Council of the United Kingdom, the Netherlands Organization for Scientific Research, and the National Research Council of Canada. 


\section{REFERENCES}

Aalto, S., Black, J. H., Johansson, L. E. B., \& Booth, R. S. 1991, A\&A, 249, 323

Aalto, S., Booth, R. S., Black, J. H., \& Johansson, L. E. B., 1995, A\&A, 300, 369

Becker, R., \& Freudling, W. 1991, A\&A, 251, 454

Braine, J., Combes, F., \& van Driel, W., 1993, A\&A, 280, 451

Casoli, F., Dupraz, C., Combes, F., \& Kazès, I. 1991, A\&A, 251, 1

Combes, F., Casoli, F., Encrenaz, P., Gerin, M., \& Laurent, C. 1991, A\&A, 248, 607

Dickman, R. L., Snell, R. L., \& Schloerb, F. P., 1986, ApJ, 309, 326

Evans, N. J. \& Lada, E. A. 1991, in Fragmentation of Molecular Clouds and Star Formation, eds. E. Falgarone, F. Boulanger, \& G. Duvert (Boston: Kluwer), 293

Garcia-Burillo,S., Guelin, M., \& Cernicharo, J., 1993, A\&A, 274, 123

Gierens, K. M., Stutzki, J., \& Winnewisser, G. 1992, A\&A, 259, 271

Gordon, M. A., \& Burton, W. B. 1976, ApJ, 208, 346

Güsten, R., Serabyn, E., Kasemann, C, Schinkel, A., Schneider, G., Schulz, A., \& Young, K., 1993, ApJ, 402, 537

Hunter, D. A., \& Massey, P., 1990, AJ, 99, 846

Irwin, J. A., \& Avery, L. W., 1992, ApJ, 388, 328

Kenyon, S. J., Hartmann, L. W., Strom, K. M., \& Strom, S. E., 1990, AJ, 99, 869

Lada, E. A., DePoy, D., Evans, N. J., \& Gatley, I, 1991, ApJ, 371, 171

Langer, W. D., \& Penzias, A. A. 1990, ApJ, 357, 477

McCaughrean, M. J., \& Stauffer, J. R., 1994, AJ, 108, 1382

Sakamoto, S., Hayashi, M., Hasegawa, T., Handa, T., \& Oka, T., 1994, ApJ, 425, 641 
Sakamoto, S., 1996, ApJ, 462, 215

Sanders, D. B., Scoville, N. Z., \& Solomon, P. M. 1985, ApJ, 289, 373

Sanders, D. B., Scoville, N. Z., Tilanus, R. P. J., Wang, Z., \& Zhou, S., 1993, in Back to the Galaxy, eds. S. S. Holt \& F. Verter, (New York: AIP), 311

Scoville, N. Z., \& Solomon, P. M., 1974, ApJ, 187, L67

Solomon, P. M., Scoville, N. Z., \& Sanders, D. B. 1979, ApJ, 232, L89

Stutzki, J., \& Güsten, R. 1990, ApJ, 356, 513

Thornley, M. D., \& Wilson, C. D., 1994, ApJ, 421, 458

Tilanus, R. P. J., Tacconi, L. J., Sutton, E. C., Zhou, S., Sanders, D. B., Wynn-Williams, C. G., Lo, K. Y., \& Stephens, S. A., 1991, ApJ, 376, 500

Turner, B. E., 1984, Vistas in Astronomy, 27, 303

Vilchez, J. M., Pagel, B. E. J., Diaz, A. I., Terlevich, E., \& Edmunds, M. G., 1988, MNRAS, 235,633

Wall, W. F., Jaffe, D. T., Bash, F. N., \& Israel, F. P., 1991, ApJ, 380, 384

White, R. E., 1977, ApJ, 211, 744

Wilking, B. A., Lada, C. J., \& Young, E. T., 1989, ApJ, 340, 823

Wilson, C. D., 1995, ApJ, 448, L97

Wilson, C. D., \& Matthews, B. C., 1995, ApJ, 455, 125

Wilson, C. D., \& Scoville, N., 1990, ApJ, 363, 435

Wilson, C. D., \& Scoville, N., 1991, ApJ, 370, 184

Wilson, C. D., \& Scoville, N., 1992, ApJ, 385, 512 
Wilson, C. D., \& Walker, C. E., 1994, ApJ, 432, 148 
Fig. $\quad 1 .-{ }^{12} \mathrm{CO} \mathrm{J}=2-1,{ }^{13} \mathrm{CO} \mathrm{J}=2-1$, and ${ }^{12} \mathrm{CO} \mathrm{J}=3-2$ spectra for seven giant molecular clouds in M33. The spectra are binned to $1 \mathrm{~km} \mathrm{~s}^{-1}$ resolution and scaled to the main beam temperature scale. All spectra refer to a $22^{\prime \prime}$ beam; the higher resolution ${ }^{12} \mathrm{CO} \mathrm{J}=3-2$ data have been convolved to this larger beam. The ${ }^{13} \mathrm{CO} \mathrm{J}=2-1$ spectrum has been scaled up by a factor of 3 .

Fig. 2.- (a) A typical large velocity gradient solution for the average CO line ratios for six giant molecular clouds in M33. The lines indicate the observed $\pm 1 \sigma$ values for the line ratios of: ${ }^{12} \mathrm{CO} /{ }^{13} \mathrm{CO} \mathrm{J}=1-0(9.7 \pm 0.6$, dashed line $) ;{ }^{12} \mathrm{CO} /{ }^{13} \mathrm{CO} \mathrm{J}=2-1(7.3 \pm 0.5$, dotted line); ${ }^{12} \mathrm{CO} \mathrm{J=3-2/J=2-1}(0.69 \pm 0.06$, solid line $)$. Acceptable values of molecular hydrogen density $n_{H_{2}}$ and ${ }^{12} \mathrm{CO}$ column density per unit velocity $N(C O) / \Delta V$ for a kinetic temperature $T_{K}=20$ are found where all three sets of lines intersect. This figure shows solutions for an adopted $\left[{ }^{12} \mathrm{CO}\right] /\left[{ }^{13} \mathrm{CO}\right]$ abundance ratio of 50; slightly warmer $(30 \mathrm{~K})$ solutions are possible if we adopt an abundance ratio of 30. (b) A similar large velocity gradient solution showing the lowest kinetic temperature solution $(100 \mathrm{~K})$ for the cloud NGC 604-2 located in the giant HII region NGC 604. The values plotted for the ${ }^{12} \mathrm{CO} /{ }^{13} \mathrm{CO} \mathrm{J}=1-0$ and $\mathrm{J}=2-1$ ratios are the same as in (a); for the ${ }^{12} \mathrm{CO} \mathrm{J}=3-2 / \mathrm{J}=2-1$ ratio, the plotted values are $1.07 \pm 0.16$, where the uncertainty includes the absolute calibration uncertainty.

Fig. 3.- Large velocity gradient solutions with a kinetic temperature of $15 \mathrm{~K}$ for three different assumed values of the $\left[{ }^{12} \mathrm{CO}\right] /\left[{ }^{13} \mathrm{CO}\right]$ abundance ratio. This figure demonstrates the existence of solutions with different values of the abundance ratio but the same density, kinetic temperature, and ${ }^{13} \mathrm{CO}$ column density. The lines indicate the observed $\pm 1 \sigma$ values for the line ratios of: ${ }^{12} \mathrm{CO} /{ }^{13} \mathrm{CO} \mathrm{J}=1-0\left(9.1,10.3\right.$, dashed line); ${ }^{12} \mathrm{CO} /{ }^{13} \mathrm{CO} \mathrm{J}=2-1(6.8,7.8$, dotted line $) ;{ }^{12} \mathrm{CO} \mathrm{J}=3-2 / \mathrm{J}=2-1$ (0.63,0.75, solid line). The vertical solid line indicates the common density solution of $8300 \mathrm{~cm}^{-3}$. (a) $\left[{ }^{12} \mathrm{CO}\right] /\left[{ }^{13} \mathrm{CO}\right]=30$. (b) $\left[{ }^{12} \mathrm{CO}\right] /\left[{ }^{13} \mathrm{CO}\right]=50$. (c) $\left[{ }^{12} \mathrm{CO}\right] /\left[{ }^{13} \mathrm{CO}\right]=70$. 
Table 1. Log of JCMT Observations

\begin{tabular}{|c|c|c|c|c|c|c|c|}
\hline \multirow[b]{2}{*}{ Cloud } & \multirow[b]{2}{*}{$\begin{array}{c}\alpha(1950) \\
\left(\begin{array}{lll}h & m s\end{array}\right)\end{array}$} & \multirow[b]{2}{*}{$\begin{array}{c}\delta(1950) \\
\left({ }^{\circ} \prime \prime \prime \prime\right)\end{array}$} & \multicolumn{3}{|c|}{${ }^{12} \mathrm{CO}$ and ${ }^{13} \mathrm{CO} \mathrm{J}=2-1$ data } & \multicolumn{2}{|c|}{$\underline{12} \mathrm{CO} \mathrm{J}=3-2$ data } \\
\hline & & & Date $^{a}$ & $\begin{array}{c}\text { Time } \\
\left({ }^{12} \mathrm{CO}, m\right)\end{array}$ & $\begin{array}{c}\text { Time } \\
\left({ }^{13} \mathrm{CO}, m\right)\end{array}$ & Date $^{b}$ & $\begin{array}{l}\text { Time } \\
(m)\end{array}$ \\
\hline MC 1 & 01:31:03.0 & $30: 23: 56$ & $94 / 7 / 17^{\mathrm{c}}$ & 18 & 72 & $95 / 8 / 1$ & 60 \\
\hline MC 13 & $01: 31: 10.3$ & $30: 20: 40$ & $94 / 7 / 16-17^{\mathrm{c}}$ & 24 & 62 & $95 / 8 / 1$ & 60 \\
\hline MC 19 & $01: 31: 13.4$ & $30: 23: 52$ & $93 / 8 / 1$ & 24 & 60 & $95 / 8 / 1$ & 60 \\
\hline MC 20 & $01: 31: 10.9$ & $30: 25: 24$ & $93 / 8 / 1$ & 24 & 72 & $95 / 7 / 31^{\mathrm{d}}$ & 48 \\
\hline MC 32 & $01: 30: 51.8$ & $30: 23: 52$ & $93 / 7 / 30$ & 30 & $42^{\mathrm{e}}$ & $95 / 8 / 1$ & 60 \\
\hline NGC 604-2 & $01: 31: 44.2$ & $30: 31: 27$ & $95 / 7 / 31$ & 18 & 48 & $95 / 7 / 31$ & 30 \\
\hline NGC 604-4 & $01: 31: 45.1$ & $30: 30: 58$ & $93 / 7 / 31$ & 18 & 42 & $95 / 8 / 1$ & 60 \\
\hline
\end{tabular}

${ }^{a}$ Observations 1993 July 30 - August 1 obtained with the Canadian Acousto-Optical Spectrometer with $0.248262 \mathrm{MHz}$ resolution; other observations obtained with the Dutch Autocorrelation Spectrometer (DAS) with $0.3125 \mathrm{MHz}$ resolution.

${ }^{\mathrm{b}}$ Observations obtained with DAS with $0.078125 \mathrm{MHz}$ resolution.

${ }^{\mathrm{c}}$ Telescope efficiency was $70 \%$ of normal.

${ }^{\mathrm{d}}$ Observations obtained with $0.3125 \mathrm{MHz}$ resolution.

e Observations obtained on 93/8/26 with $0.15625 \mathrm{MHz}$ resolution. 
Table 2. $\quad$ CO Line Ratios in Molecular Clouds in M33

\begin{tabular}{|c|c|c|c|c|c|c|}
\hline \multirow[t]{2}{*}{ Cloud } & $\mathrm{I}\left({ }^{12} \mathrm{CO} 2-1\right)$ & $\Delta V_{F W H M}$ & ${ }^{12} \mathrm{CO} \mathrm{J}=2-1$ & ${ }^{12} \mathrm{CO} \mathrm{J}=3-2$ & ${ }^{12} \mathrm{CO} \mathrm{J}=1-0^{\mathrm{a}}$ & \multirow[t]{2}{*}{$12+\log (\mathrm{O} / \mathrm{H})^{\mathrm{b}}$} \\
\hline & $\left(\mathrm{K} \mathrm{km} \mathrm{s}^{-1}\right)$ & $\left(\mathrm{km} \mathrm{s}^{-1}\right)$ & ${ }^{13} \mathrm{CO} \mathrm{J}=2-1$ & ${ }^{12} \mathrm{CO} \mathrm{J}=2-1$ & ${ }^{13} \mathrm{CO} \mathrm{J}=1-0$ & \\
\hline \multicolumn{7}{|l|}{ HII region: } \\
\hline MC 1 & $6.7 \pm 0.3$ & 8.0 & $6.9 \pm 1.0$ & $0.87 \pm 0.07$ & $9.2 \pm 2.0$ & 9.02 \\
\hline MC 13 & $6.8 \pm 0.4$ & 7.5 & $6.2 \pm 0.7$ & $0.82 \pm 0.08$ & $10.1 \pm 0.6$ & 8.83 \\
\hline MC 20 & $13.6 \pm 0.2$ & 10.5 & $7.4 \pm 0.6$ & $0.73 \pm 0.03$ & $9.0 \pm 0.4$ & 8.91 \\
\hline NGC $604-2^{\mathrm{c}}$ & $14.6 \pm 0.1$ & 11.0 & $9.9 \pm 0.6$ & $1.07 \pm 0.03$ & $11.9 \pm 2.0$ & 8.51 \\
\hline \multicolumn{7}{|c|}{ No HII region: } \\
\hline MC 19 & $8.2 \pm 0.4$ & 10.0 & $6.3 \pm 0.4$ & $0.54 \pm 0.02$ & $11.3 \pm 0.8$ & 8.98 \\
\hline MC 32 & $12.2 \pm 0.3$ & 11.0 & $8.2 \pm 1.2$ & $0.70 \pm 0.02$ & $8.9 \pm 0.9$ & 8.83 \\
\hline NGC 604-4 & $11.8 \pm 0.4$ & 9.0 & $6.4 \pm 1.0$ & $0.50 \pm 0.04$ & $7.4 \pm 0.6$ & 8.51 \\
\hline
\end{tabular}

Uncertainties are uncertainties in the mean

${ }^{a}$ From Wilson \& Walker 1994

${ }^{\mathrm{b}}$ Metallicities interpolated from Vilchez et al. 1988

${ }^{\mathrm{c}}$ This cloud is located in the giant HII region NGC 604 
Table 3. Large Velocity Gradient Solutions for M33 Clouds

\begin{tabular}{|c|c|c|c|c|}
\hline Cloud & {$\left[{ }^{12} \mathrm{CO}\right] /\left[{ }^{13} \mathrm{CO}\right]$} & $\begin{array}{l}T_{K} \\
(\mathrm{~K})\end{array}$ & $\begin{array}{c}n_{H_{2}} \\
\left(\mathrm{~cm}^{-3}\right)\end{array}$ & $\begin{array}{c}N\left({ }^{12} \mathrm{CO}\right) \\
\left(\mathrm{cm}^{-2}\right)\end{array}$ \\
\hline \multirow[t]{3}{*}{ NGC 604-2 } & 30 & $200-300$ & $2-3 \times 10^{3}$ & $20 \times 10^{17}$ \\
\hline & 50 & $100-300$ & $1-3 \times 10^{3}$ & $30-70 \times 10^{17}$ \\
\hline & 70 & $100-300$ & $1-3 \times 10^{3}$ & $50-100 \times 10^{17}$ \\
\hline with HII regions & 30 & $30-100$ & $2-6 \times 10^{3}$ & $5-9 \times 10^{17}$ \\
\hline (MC 1, MC 13, & 50 & $20-50$ & $2-7 \times 10^{3}$ & $7-20 \times 10^{17}$ \\
\hline MC 20) & 70 & $15-30$ & $3-10 \times 10^{3}$ & $7-20 \times 10^{17}$ \\
\hline without HII regions & 30 & $10-20$ & $5-30 \times 10^{3}$ & $2-4 \times 10^{17}$ \\
\hline (MC 19, MC 32, & 50 & 10 & $10-20 \times 10^{3}$ & $3 \times 10^{17}$ \\
\hline NGC 604-4) & 70 & 10 & $10 \times 10^{3}$ & $4 \times 10^{17}$ \\
\hline average of six clouds & 30 & $15-30$ & $3-10 \times 10^{3}$ & $2-6 \times 10^{17}$ \\
\hline \multirow[t]{2}{*}{ (without NGC 604-2) } & 50 & $10-20$ & $4-30 \times 10^{3}$ & $3-6 \times 10^{17}$ \\
\hline & 70 & $10-20$ & $6-20 \times 10^{3}$ & $4-6 \times 10^{17}$ \\
\hline
\end{tabular}



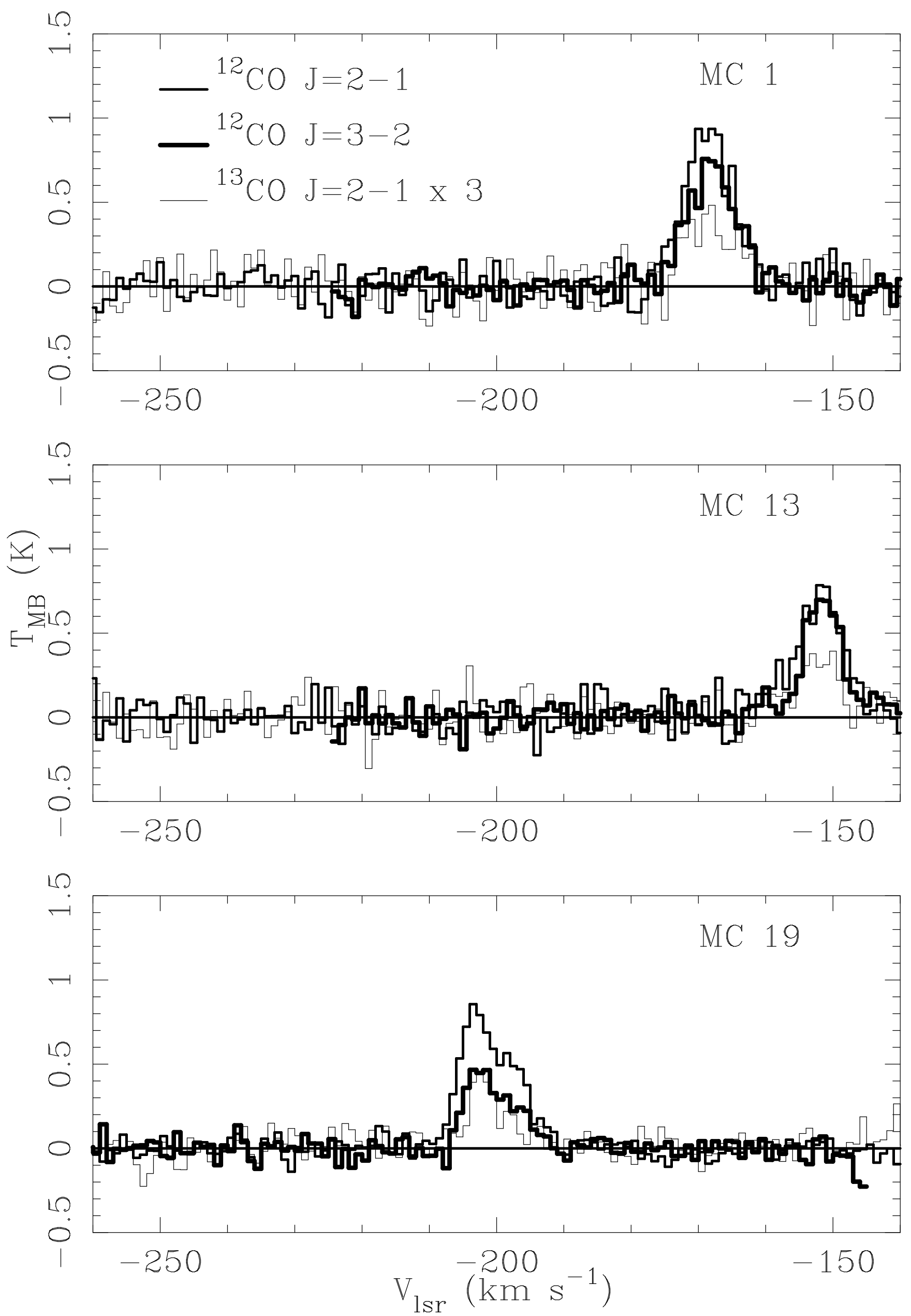

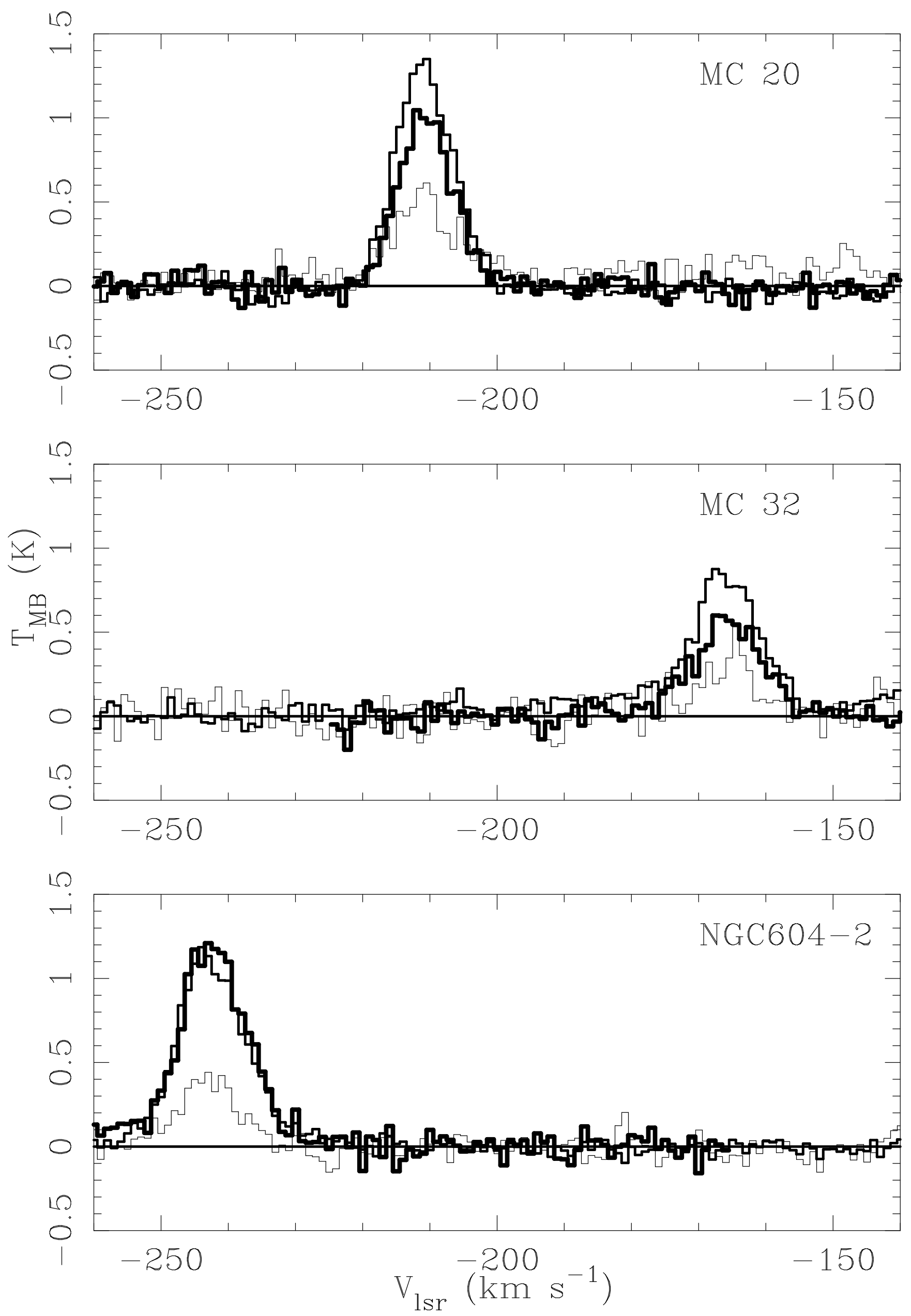


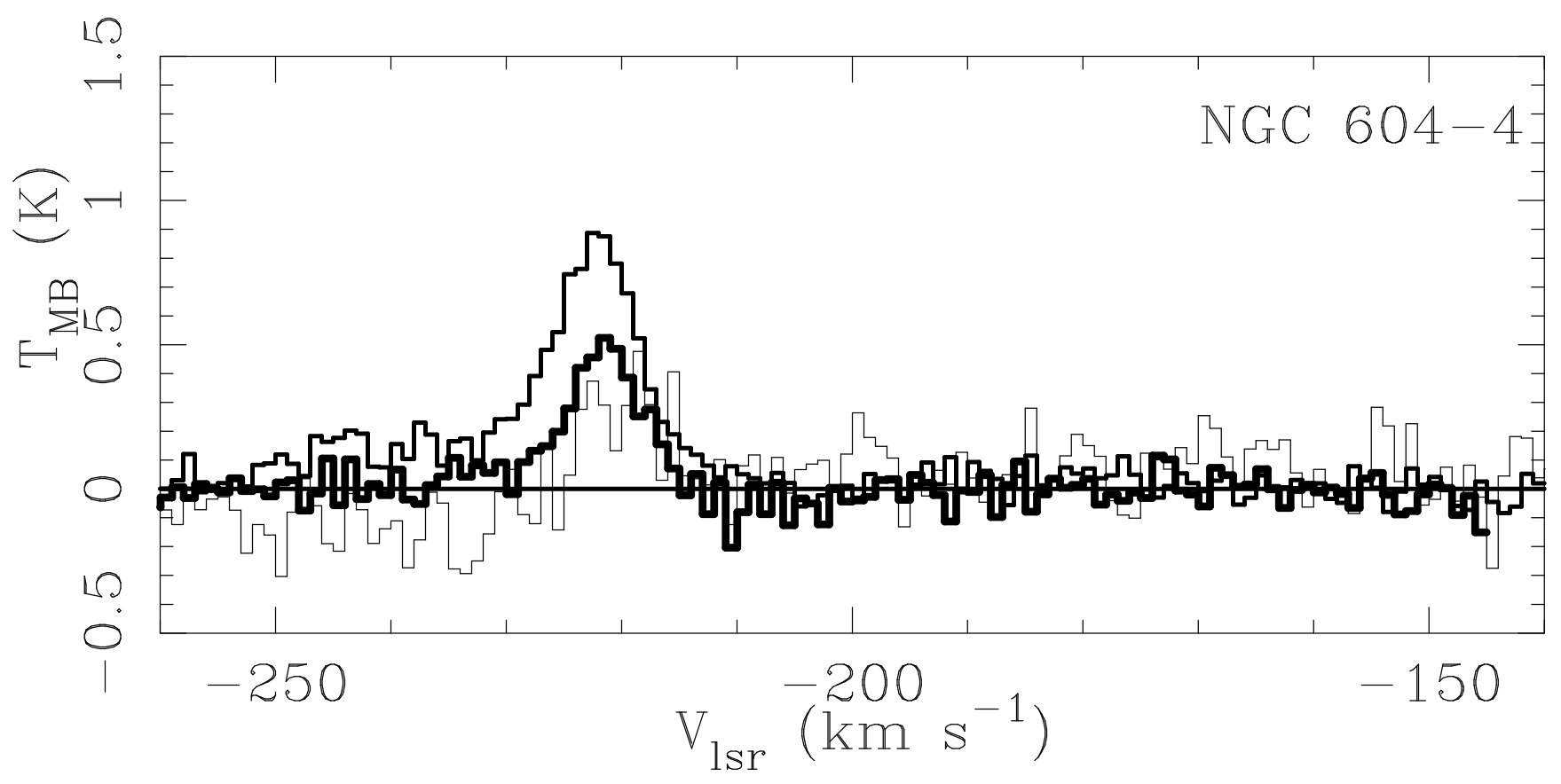




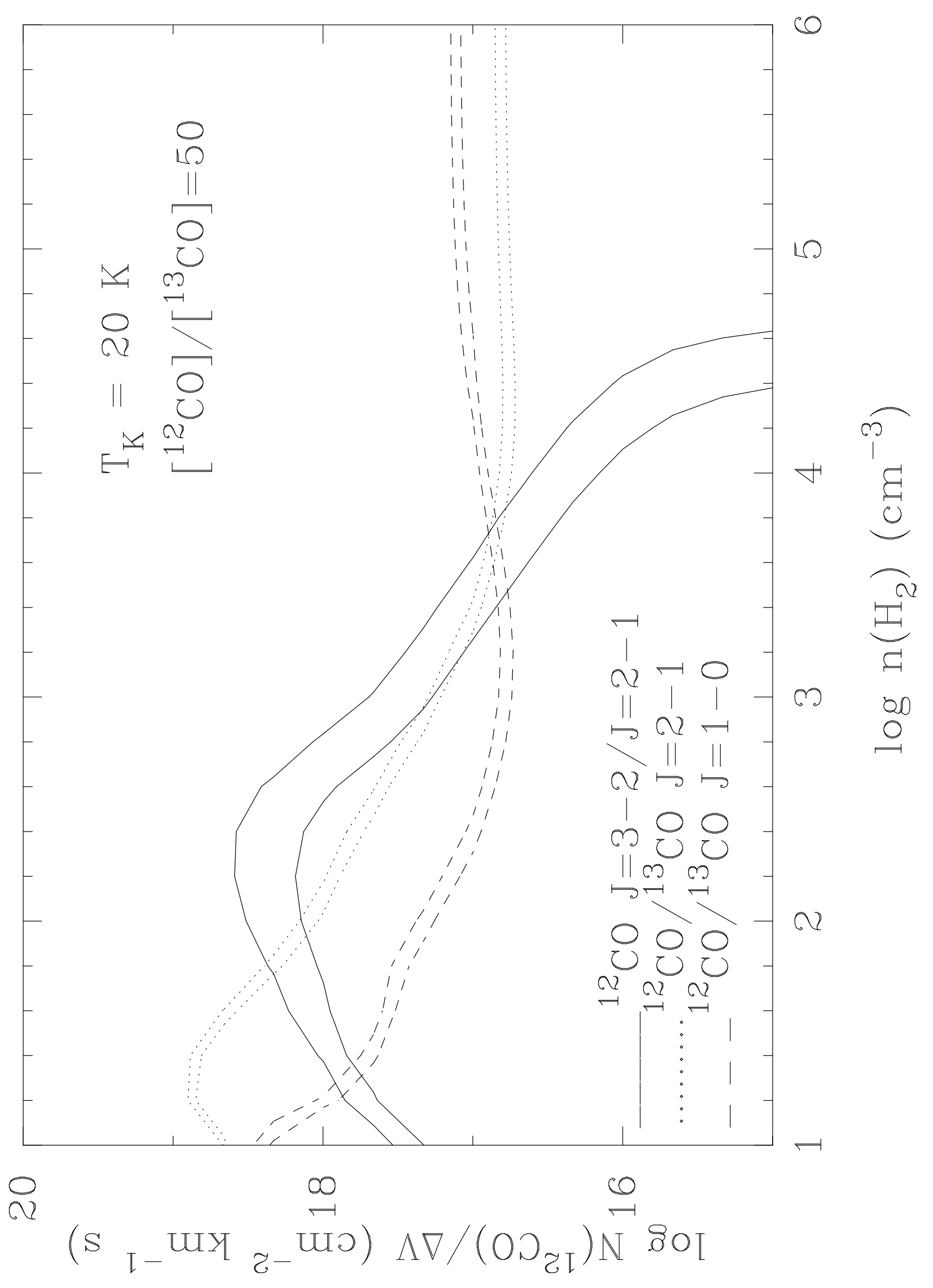




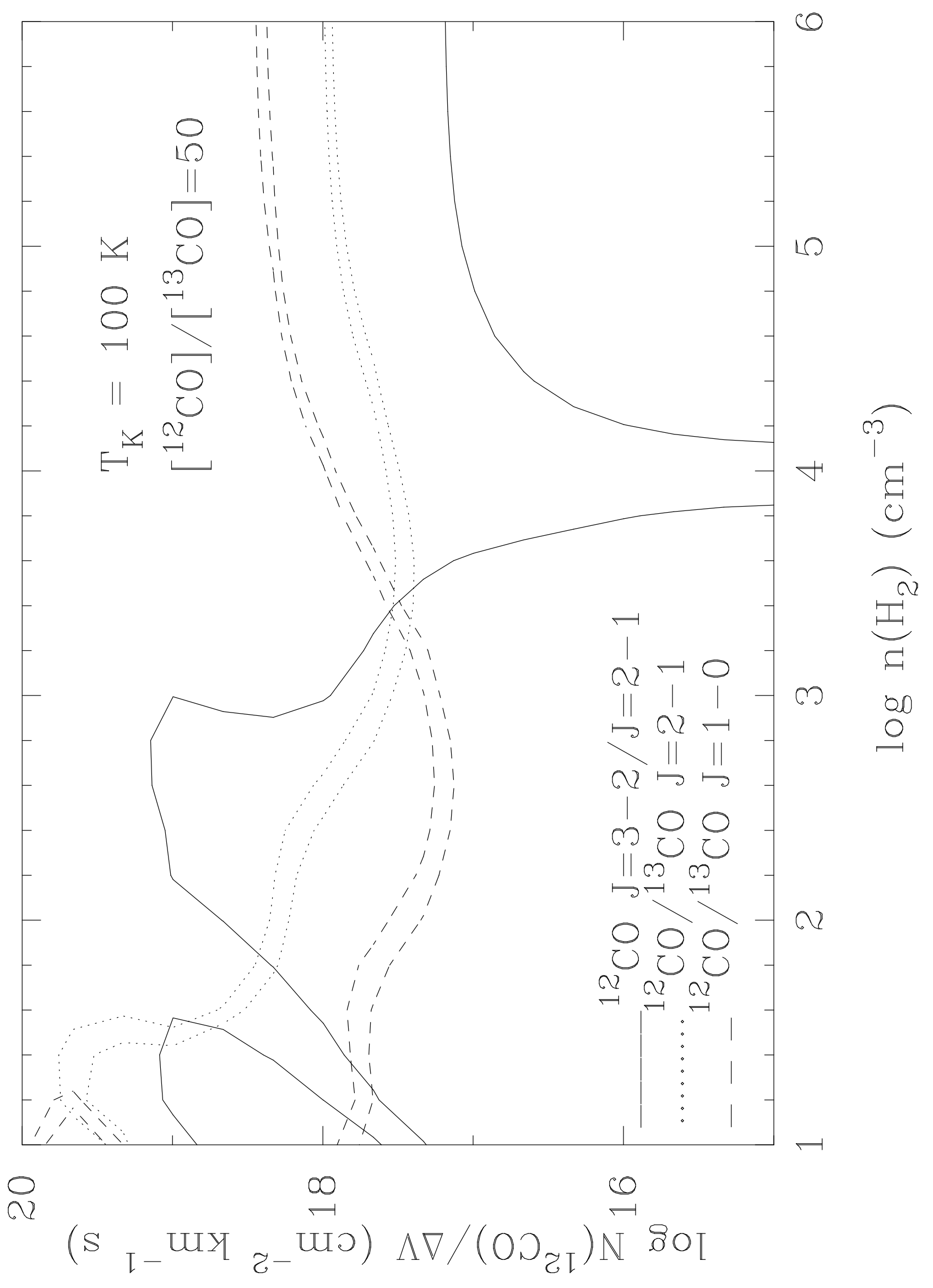




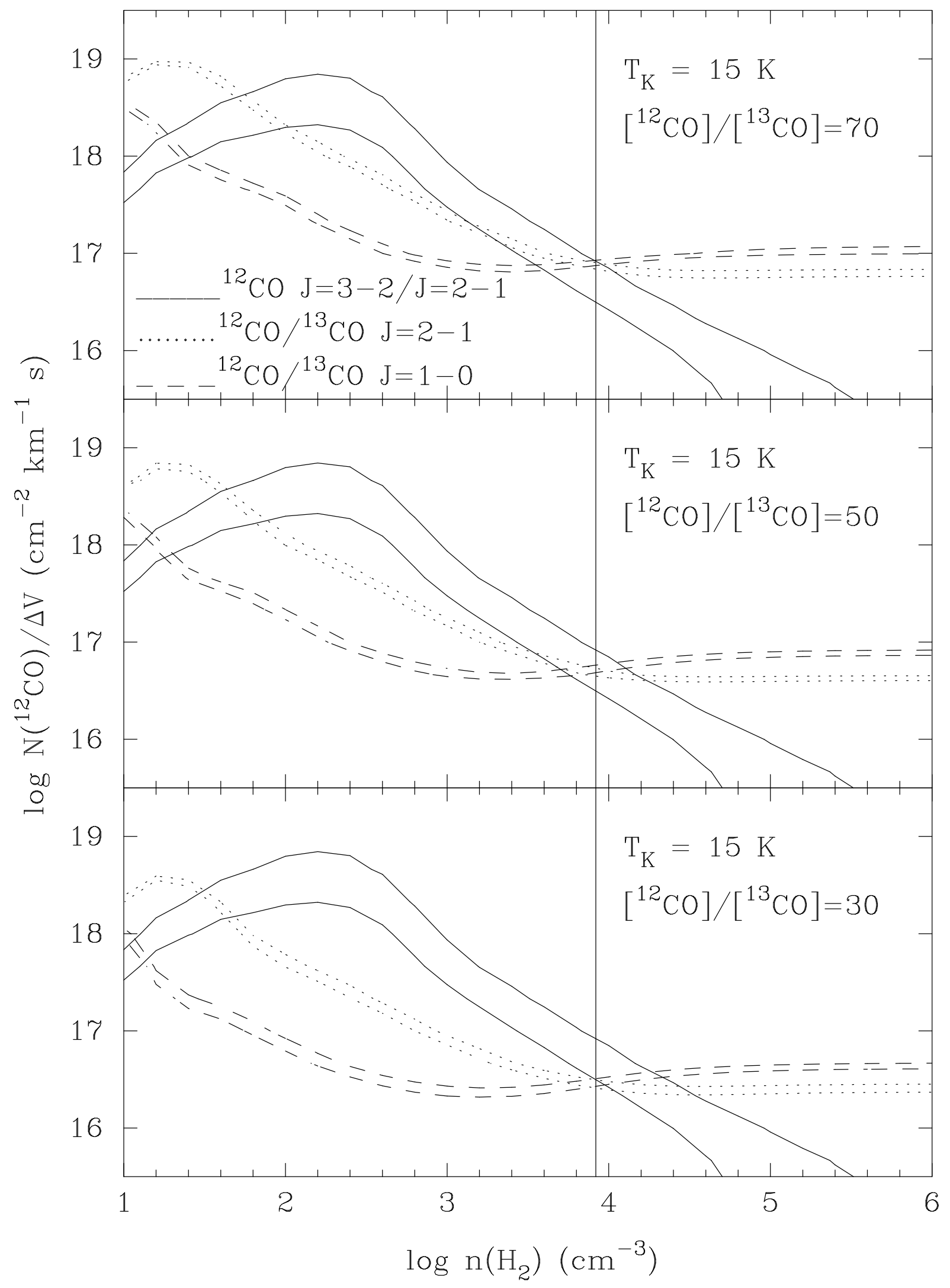

